\title{
An algorithm for cost optimization of PMU and communication infrastructure in WAMS
}

\author{
Marco A. R. S. Cruz*, Helder R. O. Rocha ${ }^{1}$, Marcia H. M. Paiva ${ }^{2}$, Marcelo \\ E. V. Segatto ${ }^{3}$ \\ Laboratory of Telecommunications at Federal University of Espirito Santo, Vitória, Brazil \\ Eglantine Camby ${ }^{4}$, Gilles Caporossi ${ }^{5}$ \\ GERAD \& HEC Montréal, Montréal, Canada.
}

\begin{abstract}
Power system state estimation relies increasingly on PMU measurements to effectively control and monitor growing and stressed transmission networks which are also affected by transient and dynamic events. High PMU cost has motivated optimal PMU placement solutions but recent works have shown the effect of communication infrastructure cost in PMU configuration. In this paper, we present a new method for the design of Wide Area Measurement Systems. A topological analysis algorithm based on the Variable Neighbourhood Search heuristic is proposed and tested in several networks, including the common IEEE test networks and the 5804-bus Brazilian transmission system. Our results show the flexibility, effectiveness, and scalability of the proposed methodology when compared with recent research presented in the literature.
\end{abstract}

\footnotetext{
This work was partially supported by CAPES-Project 88881.062156/2014-01, CNPqProjects 462477/2014-2 and 304564/2016-8 (Brazil) and by the Fondation HEC Montréal (Canada).

${ }^{*}$ Corresponding author

Email address: madrsc@yahoo.com (Marco A. R. S. Cruz*)

${ }^{1}$ helder.rocha@ufes.br

2 marcia.paiva@ufes.br

3 segatto@ele.ufes.br

${ }^{4}$ ecamby@ulb.ac.be

${ }^{5}$ gilles.caporossi@hec.ca
}

Preprint submitted to International Journal of Electrical Power and Energy SystemsSeptember 13, 2018 
Keywords: Phasor Measurement Unit (PMU), optimal PMU placement, Variable Neighbourhood Search (VNS), communication infrastructure, power system observability.

\section{Introduction}

The impacts of increasing renewable power, free access to transmission networks and deregulation of the electricity sector are changing the nature of power systems. This fact brings more stress to electrical power system operation, leading to an increasing need to improve the control and protection systems offered by Wide Area Measurement Systems (WAMS) based on Phasor Measurement Units (PMU) [1].

State Estimation (SE) processes a redundant set of measurements in order to obtain in real time a reliable estimate of the operating state of the power system. Conventionally, network state monitoring is done with conventional meters (magnitude of bus, active/reactive power flows and injections along with some voltage magnitude) from which measurements are transmitted to the $\mathrm{Su}-$ pervisory Control and Data Acquisition Systems (SCADA). With the introduction of PMUs in the 1990's, the conventional meters with Remote Terminal Units (RTUs) are now being complemented by PMUs which transmit magnitude and phase measurements to the SCADA system. The PMUs are synchronized through a Global Positioning System (GPS), which allows WAMS to be in control and operation of power systems. Another advantage is the PMU high data sampling combined with SE process that ensures quick voltage control compared to conventional measurements. However, PMUs and all related synchrophasor infrastructure deployment can be very costly [2] in large power systems, thus provoking the Optimal PMU Placement (OPP) problem on transmission networks. 
Many algorithms have been proposed to solve the OPP problem by taking into account criteria such as observability, contingencies (single PMU outage or single branch failure events), critical measurements and critical assemblies. Most of these algorithms focus on minimizing PMU costs leading to objective functions which simply optimize the number of PMUs and their placements. A few recent studies have called attention to other aspects such as Communication Infrastructure (CI), which makes a greater contribution to monitoring system costs than PMUs. In such studies, the problem is formulated in a more comprehensive way, seeking to optimize the allocation of PMUs and communication infrastructure for a minimum total cost.

Centralized WAMS architecture (Figure 1) is very suitable due to an efficient use of control elements, lower cost, good coordination of alarms and event management and lower latency. This performance is a consequence of a smaller number of Phasor Data Concentrators (PDCs) although it is exposed to a greater probability of failure than a distributed architecture [3].

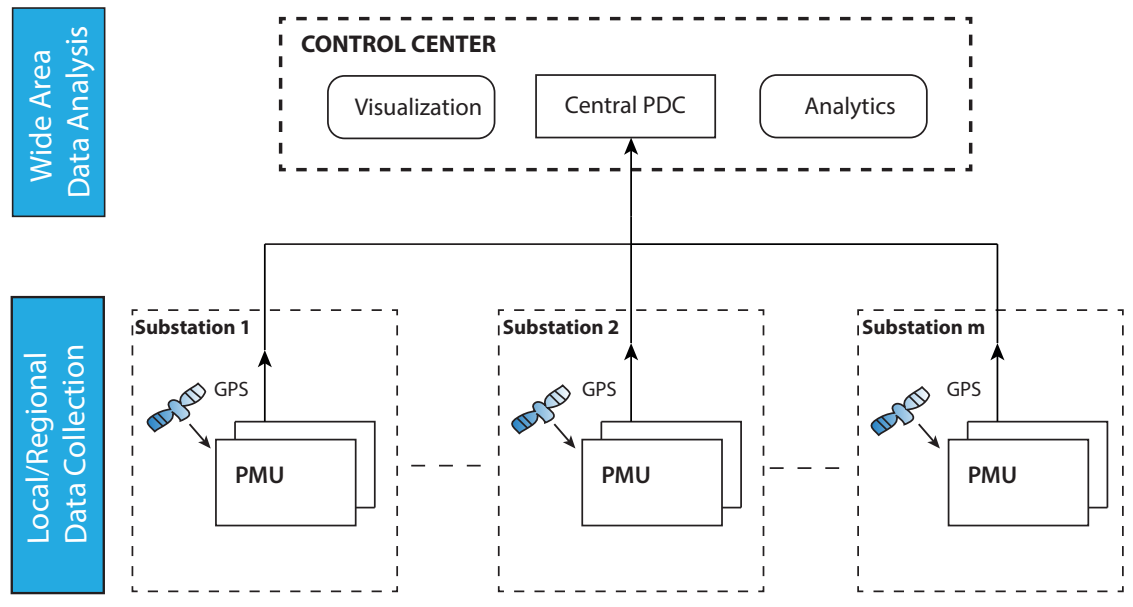

Figure 1: Centralized WAMS Architecture.

Among the transmission media for communication in smart grids, dependent 
media, i.e., those which are part of a power system and are owned by independent system operators (ISO) such as Power Line Communication (PLC), AllDielectric Self Supporting (ADSS) and Optical Power Ground Wire (OPGW), are preferred due to lower latency compared with other medias [4]. However, OPGW stands out due to the advantages of high channel capacity and transfer rate, low transmission losses and immunity to electromagnetic interference [5, 6]. Furthermore, the CI can be co-optimally designed in conjunction with power system planning problems.

\subsection{Contribution}

This paper proposes new methods and algorithms for the OPP problem based on a topological observability analysis and for simultaneously optimizing PMU allocation and communication network leading to a minimum total cost in WAMS. Other costs besides PMU and CI are outside the scope of this work. The algorithm for the latter problem is derived from the first and include Dijkstra's single-source shortest-path and Kruskal minimum spanning tree algorithms to achieve a minimum total cost. To construct both algorithms, a Variable Neighbourhood Search (VNS) heuristic [7 is implemented. To the best of our knowledge, it is the first time that a VNS heuristic is applied to the OPP problem.

The problems above are related to graph theory concepts such as Dominating Set and Connected Dominating Set [8, 9]. These concepts have motivated the construction of a new metric, named here Dominance, which plays a fundamental role in improving the efficiency of the proposed algorithms in combination with other known graph theory metrics. Our algorithms have proved to be flexible as they incorporate various cost parameters and contingencies. Additionally, they are scalable when generating results from small networks to large and complex electrical transmission networks and show good efficiency when 
the results are compared with other works.

This paper proposes an optimization of costs on a centralized WAMS supported by OPGW transmission media, in considering the costs of the switches. These are understood as electro-optical and/or optic-optical converters integrated into an Add Drop Multiplexer (ADM), which enable PMU connection to the OPGW communication network or allow the various OPGW branches arriving on a bus to form a communication network node.

\subsection{Related work}

There are several works on Optimal PMU Placement (OPP) which have been developed over approximately 25 years 10 . These algorithms are classified according to the type of observability analysis, numerical or topological [11]. Despite the fact that topological methods are faster and more suitable for large systems, some numerical algorithms have been shown to be more efficient in allocating PMUs [12].

All these algorithms can still choose to use deterministic 13 or metaheuristic methods [14. Although metaheuristic algorithms have advantages in computational time (especially in large systems) and in the ability to deal with conditions such as single PMU outage, topological variation, critical measurements and areas of interest, trapping in local minima is still a possible problem [15].

The consideration of realistic costs and practical constraints related to substations have either led to different approaches beyond the OPP problem - such as the Optimal Substation Coverage Algorithm [16] - or have forced the OPP problem to consider the cost of upgrading substations for PMU placement [17.

Communication Infrastructure (CI) is also another factor which is influencing the OPP problem beyond conventional PMU cost-minimization paradigm. In this sense, the problem is modeled as a total cost minimization problem [4, taking into account both PMU and CI costs and using a genetic algorithm to 
confirm that the lowest total cost does not always correspond to the smallest number of PMUs. The paper [18] has a similar approach and proposes a multiobjective algorithm to address PMU, PDC and CI placement, simultaneously.

In [5], this previous approach is explored in greater depth and other aspects and devices involved in communication infrastructure are detailed. Additionally, that work considers contingency conditions and pre-existence of some PMUs and communication cables in certain parts of the network. In the same way, [19] considered CI cost and several contingency conditions in a binary gravitational search algorithm, which showed better performance than in the previous work.

Still, the same approach to total cost optimization is highlighted in [20] and a realistic cost-effective model is presented for optimal PMU placement which considers practical and unaccounted cost implications based on a reallife project. The results confirmed that a minimal number of PMUs does not necessarily indicate minimum financial implications of the OPP project.

As an alternative to the existing fiber-based communication infrastructure, microwave communication technology was considered in an OPP problem [21] with focus on minimizing the propagation delay.

The remaining sections of this paper are organized as follows. Section 2 gives an introduction to the fundamental concepts used throughout the paper. Section 3 presents the OPP problem and a VNS-based heuristic to solve it. Section 4 extends the problem to a total cost objective-function and details the algorithm implementation. Section 5 provides simulation results and finally, the conclusions are drawn in Section 6 .

\section{Technical Background}

This section covers the theoretical fundamentals used throughout this pa-

per: the basis of Metaheuristic Variable Neighbourhood Search, graph theory 
concepts and algorithms, and topological observability analysis.

\subsection{Metaheuristic Variable Neighbourhood Search}

The Variable Neighbourhood Search (VNS) is a metaheuristic introduced by [7] that counts on several successful applications in solving combinatorial and global optimization problems 22. VNS relies on systematic changes in the vicinity for local search. This metaheuristic is characterized by simplicity and efficiency.

VNS is based on a variable neighbourhood method which exploits neighbourhoods progressively further away from the current solution and moves to another solution if it is better than the previous one. This metaheuristic has three stages to search for new solutions: (1) Initial solution, (2) Shaking (or perturbation), which allows a progressive varying of the neighbourhood of the current solution; and (3) Local search (or descent), which is a local search procedure. The shaking stage is necessary to move the current solution away from a local optimum, and it is parameterized by the shaking amplitude $k$, which changes the current solution more and more as it increases. Each value of $k$ corresponds to a different neighbourhood $N^{k}$. The search can become more global and diverse when there is no progress in the solution. If the local search can improve the solution prior to a perturbation, then the new solution is adopted and the perturbation is resumed with value $k=1$. This process is iterative and continues until a stopping criterion is reached.

The basic structure of the VNS is presented below although there are some variations of the algorithm [7]:

\section{Basic VNS Algorithm}

Function VNS $\left(x, k_{\max }, t_{\max }\right)$

1: Repeat

2: $\quad k \leftarrow 1$ 


$$
\begin{aligned}
& \text { 3: } \quad \text { Repeat } \\
& \text { 4: } \quad x^{\prime} \leftarrow \text { Shake }(x, k) \\
& \text { 5: } \quad x^{\prime \prime} \leftarrow \text { FirstImprovement }\left(x^{\prime}\right) \\
& \text { 6: } \quad x \leftarrow \text { NeighbourhoodChange }\left(x, x^{\prime \prime}, k\right) \\
& \text { 7: } \quad \text { Until } k=k_{\max } ; \\
& \text { 8: } \quad t \leftarrow \text { CpuTime }() \\
& \text { 9: } \text { Until } t>t_{\max } ;
\end{aligned}
$$

\subsection{Graph Theory Concepts and Algorithms}

A graph $G=(V, E)$ is a set of $|V|$ vertices along with a set of $|E|$ edges connecting those vertices. A graph with $N=|V|$ vertices can be represented by an adjacency matrix $A=\left[a_{i j}\right]_{N \times N}$, where $a_{i j}=1$ if there is an edge connecting vertex $i$ to vertex $j$, and 0 if otherwise. A subgraph is a subset obtained by removing any set of vertices or edges from the original graph. The degree of a vertex $i$ is the number of edges incident to $i$.

Betweenness Centrality (BWC) or simply Betweenness is a measurement of how significant a vertex is in facilitating communication between any two vertices in the network by means of the shortest paths.

To find shortest paths between vertices in a graph $G=(V, E)$ with nonnegative edge weights, the single-source shortest path Dijkstra algorithm is used. The running time of this algorithm is $O(|V| \log |V|)$ 23].

The spanning tree of a graph $G=(V, E)$ consists of a connected acyclic subgraph $T=\left(V, E^{\prime}\right)$ which contains all the vertices of $G$ and a subset $E^{\prime} \subset E$ of its edges. To find a minimum-spanning-tree, that is, a spanning tree minimizing the sum of edge weights, the Kruskal Algorithm is used. It can easily be made to run in time $O(|E| \log |V|)[23$.

The problem proposed in this paper is closely related to the minimum connected dominating set problem [24]. A dominant set (DS) of a graph $G=(V, E)$ 
is a subset $D \subset V$ such that each element $v$ of $V$ is either in DS or is connected to a vertex in DS. In the latter case, $v$ is said to be covered by a vertex in DS. A connected dominant set (CDS) is a connected subgraph induced by vertices of $\mathrm{D}$. The problem related to these concepts is the cardinality minimization of DS or CDS, with some applications in wireless network problems [25].

\subsection{Topological observability analysis}

In a topological observability analysis, a power system is represented by a topological graph, where the vertices represent the network buses and the edges represent the network branches connecting the buses. Topological observability analysis is defined as the existence of at least one spanning measurement tree of full rank in a network [26]. This tree connects all observable buses and branches which can be observed by direct measurements or calculations. The existence of this tree is commonly assessed by the following rules [27:

1. Assign with direct voltage phasor measurement and direct current phasor measurement of incident lines the buses with PMUs;

2. If voltage and current phasors at one end of a branch are known, then the unknown voltage phasor at the other end of the branch can be calculated (called a pseudo-measure) through Ohm's Law;

3. A current phasor of a branch can be calculated (pseudo-measure) if voltage phasors of both ends of this branch are known;

4. If all line current phasors incident to a zero-injection bus (ZIB) are known except one, the current phasor of the unknown one can be calculated through Kirchhoff's Circuit Law equations. ZIB is a bus in which the net power injection is zero. This is used as pseudo information to make a system observable with a lesser number of PMUs compared to the case when information of ZIBs is not considered; 
5. If there is an unknown ZIB bus and voltage phasors of its adjacent buses are all known, then it is possible to calculate the voltage phasor of the ZIB bus.

\section{Proposed Method for Optimal PMU Placement Problem}

This section presents the OPP problem formulation considering only the PMU cost, and the proposed method to solve it. The formulation takes into account the following constraints: observability in normal condition and in N-1 contingency conditions, such as single PMU or branch outage.

A new metric designed specifically for the OPP problem, called Dominance Centrality, is introduced here, and a VNS-based algorithm is proposed to solve this problem.

\subsection{PMU Placement Problem Formulation}

For an $N$ bus system, the optimal PMU placement problem is formulated as follows:

$$
\operatorname{Min}\left[\sum_{i=1}^{N} c p_{i} \cdot x_{i}\right]
$$

$$
\text { s.t. } A \cdot X \geq b \text {, }
$$

where:

$c p_{i}$ is the cost of placing a PMU at bus $i$;

$A$ is the adjacency matrix;

$b=\left[\begin{array}{lllll}1 & 1 & 1 & \ldots & 1\end{array}\right]^{T}$ is a unit vector of length $N$;

$X$ is the binary decision variable vector for PMU placement, which entries $x_{i}=1$ if a PMU is placed at bus $i$, and zero if otherwise.

Observability requirement is attained by Constraint (2) under normal conditions. However, this constraint has to be replaced by Equations (3) or (4) if 
observability is imposed under single PMU or single branch outage conditions, respectively.

$$
\begin{gathered}
A \cdot X \geq\left[\begin{array}{llll}
2 & 2 & 2 & \ldots
\end{array}\right]^{T} \\
A_{j} \cdot X \geq\left[\begin{array}{lllll}
1 & 1 & 1 & \ldots & 1
\end{array}\right]^{T}, \quad j=1,2, \ldots, N_{t o p}
\end{gathered}
$$

In the latter equation, $A_{j}$ is a new adjacency matrix, slightly different from the original matrix $A$ reflecting topology change inflicted by the branch outage. $N_{\text {top }}$ is the number of possible scenarios of single branch outage.

The optimal PMU placement problem can also be formulated in terms of minimizing the dominating set (DS) of the graph representing the power network 24]. The graph theory terms relate to system observability in the following way:

- The vertices of a DS define the buses where PMUs are allocated;

- A bus that is covered (observable) is a bus with an allocated PMU or adjacent to a bus with a PMU; and

- The vertices of a DS compose a feasible solution for PMU placement thereby guaranteeing system observability.

Therefore, the problem of finding the buses to allocate PMUs on a power network ensuring system observability corresponds to the problem of finding a minimum DS for the graph that represents the power network.

\subsection{Dominance Centrality, a new metric for DS}

Degree Centrality is largely used in topological algorithms as the main searching criterion for OPP problems [9, 28, However, this strategy is not 
very efficient in achieving a minimal number of PMUs due to the fact that some vertices with high degrees could cover (or share) the same set of adjacent vertices. To avoid or diminish multiple covering probability, the searching process should consider vertices with high degree but connected to neighbourhood vertices with low degrees. These informations are embedded together in a new metric called here Dominance Centrality (dom). The Dominance Centrality of a vertex $v_{i}, \operatorname{dom}\left(v_{i}\right)$, is defined as follows:

$$
\operatorname{dom}\left(v_{i}\right)=\operatorname{deg}\left(v_{i}\right)-\operatorname{adn}\left(v_{i}\right),
$$

where $\operatorname{deg}\left(v_{i}\right)$ is the degree of vertex $v_{i}$, and $a d n\left(v_{i}\right)$ is the average degree of all neighbourhood vertices of $v_{i}$, which is formally defined in Eq. (6) as follows:

$$
\operatorname{adn}\left(v_{i}\right)=\frac{\sum_{j=1}^{\operatorname{deg}\left(v_{i}\right)} \operatorname{deg}\left(v_{j}\right) \cdot a_{i j}}{\operatorname{deg}\left(v_{i}\right)},
$$

where each $v_{j}$ is a vertex adjacent to $v_{i}$.

\subsection{Proposed Optimal PMU Placement Algorithm}

This section describes the VNS-based heuristic proposed to solve the OPP problem defined in the previous section. To allow comparisons with other works, PMU unit cost $c p_{i}$ is taken as a constant value in the objective-function (1) stated previously, with the same constrained conditions as stated before.

The VNS is divided into three stages: (1) Initial solution, (2) Shaking and (3) Local search (descent). These stages can be adapted to each formulation of the proposed problems. 


\subsubsection{Normal operating condition}

Initial Solution: The adjacency matrix of the network, and the degree and Dominance of all its buses (vertices) are calculated and $33 \%$ of the buses are selected with the highest Dominance value for PMU allocation (dominant vertex). This percentage value is based on the work [29, in which the authors state that PMUs should be allocated to about one-quarter to one-third of the system buses to meet observability criteria. Although this is not true for many graphs, this range is used here as a reference to build an initial solution. Additionally, PMUs are assigned to all buses that are adjacent to terminal buses. Finally, a check is performed to identify which buses are not yet observed by PMUs and in such cases, PMUs are allocated to these buses.

Shaking: This stage consists of disturbing the current solution and moving it away from local minima by allocating more PMUs to other buses. In this way a new solution is obtained which can be improved by a subsequent local search. The number of PMUs to be introduced is determined by a factor that is proportional to the size of the network and the selection of the buses is done in parameterized $(k)$ and probabilistic ways. If the value of $k$ is low, there is a greater probability that buses with higher Dominance values are selected to allocate new PMUs. If $k$ increases, buses with lower Dominance value are selected, i.e., the solution (the neighbourhood) is increasingly varied. The increase of $k$ always happens when the solution can not be improved in the previous local search iteration. When the local search can improve the solution, the disturbance resumes with $k=1$.

Local Search: The local search is performed after each shaking and seeks to reduce the number of PMUs. PMUs are sequentially eliminated from buses with lowest to highest Dominance or inversely in alternating iteration. A PMU is only removed from a bus if it does not violate the observability constraint 
$A \cdot X \geq b$. The new solution is assumed whenever it has at least the same number of PMUs and, in this case, perturbation restarts with $k=1$.

\subsubsection{Single PMU loss and branch failure conditions}

Supporting single PMU loss is one of the robustness requirements for a monitoring system. In this condition it is ensured that in case of any single PMU failure the system remains observable. To meet this requirement each bus must be observed (or covered) by two PMUs, i.e., be adjacent to two PMU buses, or by one when the bus itself has another PMU allocated to it. This requirement does not introduce substantial changes in the base algorithm (observability under normal conditions) previously seen. For the initial solution, PMUs are allocated on all buses to ensure observability for single PMU loss. The shaking stage remains the same from the previous algorithm but it is not performed in the first iteration (the initial solution does not allow any perturbation). The local search is performed in the same way but it only accepts PMUs removal if all buses remain covered (or observable) by at least two PMUs $\left(A \cdot X \geq\left[\begin{array}{llll}2 & 2 & 2 & \ldots\end{array}\right]^{T}\right)$.

For branch failure conditions, the implementation looks very similar but the condition to remove PMUs in the local search stage is more relaxed since it depends on the branch failure scenario, i.e., a bus should be observed by two PMUs if a branch failure could affect connectivity to a bus with an allocated PMU, otherwise one PMU is sufficient (4).

\section{Proposed Method for PMU and Communication Infrastructure Cost Optimization Problem}

This section presents the problem formulation and the method proposed to optimize both PMU placement and related communication infrastructure (CI) for a minimum total cost associated. The proposed method is derived from the previous OPP algorithm discussed in Section 3 . 


\subsection{Problem Formulation}

The PMU and Communication Infrastructure Cost Optimization Problem is stated in Equations (7)- 10 :

$$
\operatorname{Min}\left[\sum_{i=1}^{N} c p_{i} \cdot x_{i}+\sum_{i=1}^{N} \sum_{j=1}^{N}\left(c f \cdot d_{i j}+c r \cdot x_{i}\right) \cdot y_{i j}\right]
$$

s.t.

$$
\begin{gathered}
A \cdot X \geq b \\
\sum_{i j \in E} y_{i j}=N-1 \\
\sum_{i j \in E: i, j \in S} y_{i j} \leq|S|-1, \forall S \subseteq V
\end{gathered}
$$

The objective function (7) aims to minimize the total cost related to PMUs (first term) and communication infrastructure (second term). The CI cost has two components. One is related to active elements, such as switches and routers, and the other one is related to OPGW deployment, which is proportional to the length of cables deployed.

Constraint (8) ensures power system observability under normal conditions if $b$ is a unit vector of length $N$. For N-1 contingency conditions, i.e., single PMU or branch outage, this constraint is replaced by respective Equations (3) or (4).

Constraints (9) and (10) ensure a tree topology for PMUs communication network. In this sense, Equation (9) imposes a number of links or edges $\left(y_{i j}\right)$ that is one unit less than the number of vertices $N$, and Equation 10 ensures there is no cycle by imposing that any subset of $S$ vertices must have at most $(|S|-1)$ edges connecting them. 
In this optimization, a centralized WAMS architecture is considered and a communication network with OPGW is optimized over the electric network.

In addition, the variables involved are defined as follows:

$\mathrm{N}$ is the number of buses in the network;

$c p_{i}$ is the cost of placing a PMU at bus $i$;

$c r$ is the cost of active devices, such as routers and switches, allocated in all buses with PMUs;

$c f$ is the OPGW cost per km;

$d_{i j}$ represents the distance between buses $i$ and $j$;

$y_{i j} \in\{0,1\}$ assumes value 1 if an existing line $(i, j)$ connecting buses $i$ and $j$ is selected to integrate the OPGW communication network;

$X$ is the binary decision variable vector for PMU placement whose elements $x_{i}$ are defined as one if a PMU is placed at bus $i$, and zero if otherwise;

$A$ is the adjacency matrix of the network.

\subsection{Proposed Method for Total Minimum Cost}

The proposed problem involves allocating a minimum set of PMUs and also minimizing the distances involved in the fiber cable communication network. This two-component objective is related to the Minimum Connected Dominating Set (MCDS) problem addressed in Graph Theory, which is in general NP-hard. Some methods solve the MCDS problem directly whereas other methods propose a two-phase strategy by first building the DS and then connecting it to get a CDS [30]. This two-phase strategy is not appropriated to the optimization problem proposed here because PMU placement and communication aspect of the synchrophasor infrastructure are intimately connected. In this way, a dual optimization solution is implemented to address two simultaneous optimizations, PMU placement and CI design, in order to minimize the overall cost. 
In the first stage of the algorithm, the VNS solves these simultaneous optimizations, allocating PMUs for a global cost optimization. The optimal CI design is guaranteed by Dijkstra Shortest path computations in the local search process. The second stage of the algorithm finds the shortest communication network that links all the PMUs previously found, which means finding a MCDS. This MCDS is determined in a weighted graph, which is somewhat different from MCDS's problems in the literature that aim to minimize the CDS cardinality. Therefore the proposed algorithm constructs a MCDS respecting this specificity. Although there are two stages, the MCDS is predefined in the first stage and the second stage is only necessary for further processing Dijkstra shortest path calculations already done in the first stage. The two stages of the proposed algorithm are described as follows.

\subsubsection{Stage I - Optimal PMU Placement}

The objective of this stage is not only to minimize costs with PMUs but also to choose the best PMU configuration that can impact more effectively the CI optimization and globally achieve the lowest total cost. Following reference [9, which showed the advantage of Betweenness Centrality in the determination of a MCDS, the present methodology proposes to combine both metrics, Dominance and Betweenness for PMU placement. Dominance is used to minimize the number of PMUs, while Betweenness Centrality seeks, at the same time, to influence the best PMU configuration that provides the lowest communication network costs.

A VNS-based heuristic is also used, which is slightly different from the one proposed for minimizing the number of PMUs in Section 3.3 . The operation of the VNS is detailed as follows:

Previous Calculations: From matrix A, the degree and the Dominance values are computed for each vertex; Betweenness of each vertex is computed from 
network distance and cable unit cost. Dijkstra algorithm is used to compute the single-shortest-paths from the bus with higher Dominance value to all remaining buses.

Initial Solution: $33 \%$ of the buses with the highest Dominance value for PMU allocation are selected. Additionally, PMUs are assigned to all buses that are adjacent to terminal buses. Finally, a check is performed to identify which buses are not yet observed by PMUs and in such cases PMUs are allocated to those buses. For single PMU outage and branch failure requirements, the initial solution is formed by placing PMUs in all buses in both cases.

Shaking: The process to disturb the current solution is the same as described in Section 3.3.1.

Local Search: The local search is performed after each shaking and seeks to reduce the number of PMUs. PMUs are sequentially eliminated from buses with lowest to highest Dominance or Betweenness. These two metrics are used alternately from one iteration to another. A PMU is only removed from a bus if it does not violate the observability constraint $A \cdot X \geq b$. The new solution is assumed whenever its related total cost is lower or at least the same from the previous solution. The total cost comprises costs with PMUs, switches and OPGW. This OPGW cost is determined using the shortest paths previously calculated. With a new solution a new perturbation restarts with $k=1$.

The flow chart of this algorithm is represented in Figure 2.

\subsubsection{Stage II - Communication Network Computation}

After optimal PMU placement, a communication network interconnecting the buses with PMUs is computed. This is related to constructing a CDS from a pre-determined DS. Kruskal Minimum Spanning Tree algorithm is used together with previously calculated Dijkstra's Tree to find the smallest communication network. The following steps are implemented: 


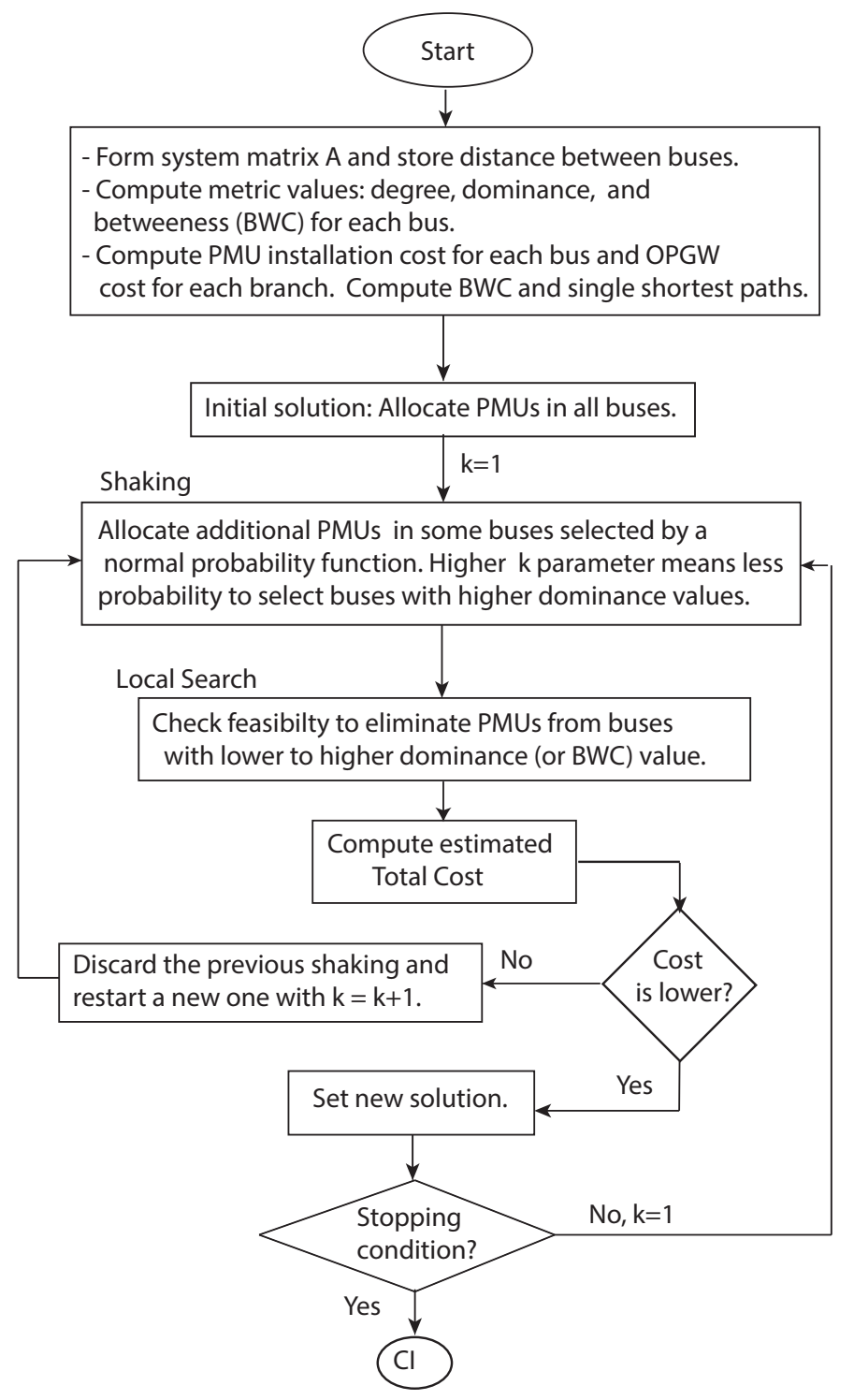

Figure 2: Flow chart of Stage I.

1. With the PMUs (DS vertices) and the single-shortest-paths previously calculated, the vertices which do not require PMUs are identified. A subgraph of the original network is obtained by removing these vertices.

2. Kruskal algorithm is applied on this subgraph to set a minimum spanning 
tree structure to the PMU communication network.

The flow chart of these steps is presented in Figure 3

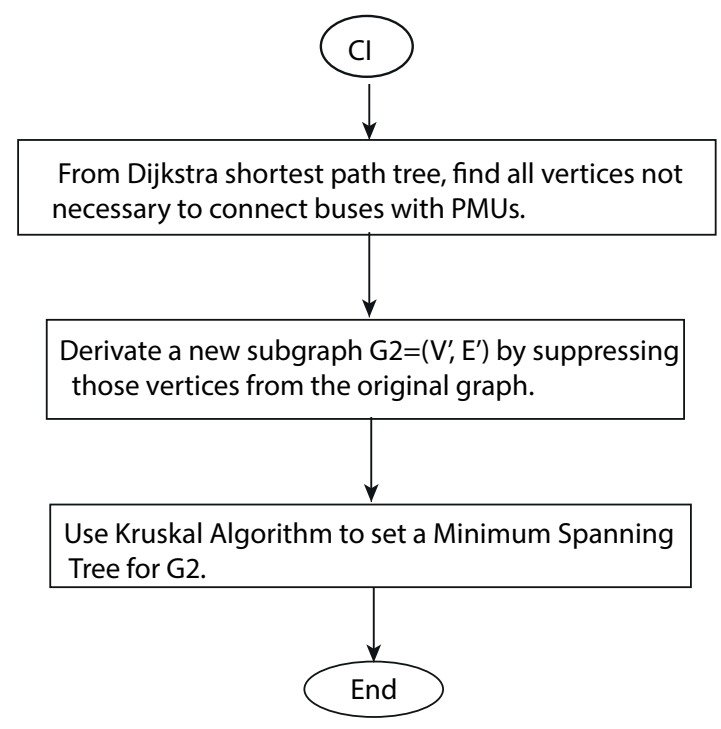

Figure 3: Flow chart of Stage II.

\section{Results and Discussion}

This section shows results of the proposed algorithms for some of the most common test transmission networks, such as IEEE 14-bus, 24-bus, 30-bus, and 118-bus. Additionally, it shows results performed for IEEE 300-bus and in 5804-bus Brazilian transmission network in order to verify the scalability of the algorithms.

\subsection{OPP Optimization Results}

Results of simulations for an optimal PMU placement problem under normal conditions (without considering ZIB) are presented in Table 11. The optimal number of PMUs required for different systems and related bus locations are 
shown. The minimum number of PMUs allocated reached the best results found in the literature [28], which is the optimal solution in this condition.

Simulations are also performed on larger networks such as IEEE 300-bus and Brazilian 5804-bus. For IEEE 300-bus, the method allocated 87 PMUs which is compared to the best result found in the literature [31. Related to the Brazilian network, $34 \%$ of buses were equipped with PMUs which is almost at the same rate for IEEE 30-bus (33\%).

Table 1: Optimal PMU Placement Results for normal operating conditions without ZIB.

\begin{tabular}{c|c|c}
\hline System & Optimal PMU locations & $\begin{array}{c}\text { No. } \\
\text { PMUs }\end{array}$ \\
\hline IEEE-14 & $2,6,7,9$ & 4 \\
\hline IEEE-24 & $2,8,10,16,21,23,24$ & 7 \\
\hline IEEE-30 & $2,4,6,9,10,12,18,23,25,29$ & 10 \\
\hline IEEE-57 & $1,5,9,12,15,17,21,23,28,30,36,40,44,48,49,52,56$ & 17 \\
\hline IEEE-118 & $\begin{array}{c}1,5,9,12,15,17,20,23,28,30,36,40,44,46,50,52,56,62, \\
63,68,71,75,77,80,85,86,90,94,102,105,110,115\end{array}$ & 32 \\
\hline
\end{tabular}

The minimum percentage of buses equipped with PMUs needed for observability depends on network topology. Generally, a dense graph (i.e., a graph whose number of edges is high compared to the number of vertices) needs less PMUs to meet observability requirements. Table 2 summarizes the percentage of PMUs allocated in the networks versus the average degree of the graph, which is one of the indicators of graph density. It can be noticed that the Brazilian Network has the highest percentage of buses with PMUs compared to other instances but it is also the least dense network (average degree 2.44).

For single branch outage operating conditions, simulations on IEEE 30-bus and 118-bus were considered with several branch failures scenarios. Results are presented in Table 3 . 
Table 2: Minimum number of PMUs versus graph average degree.

\begin{tabular}{lccccc}
\hline System & IEEE-30 & IEEE-57 & IEEE-118 & IEEE-300 & BR-5804 \\
\hline No. PMUs & 10 & 17 & 32 & 87 & 1987 \\
\% bus with PMUs & $33 \%$ & $30 \%$ & $27 \%$ & $29 \%$ & $34 \%$ \\
Average degree & 2.73 & 2.74 & 3.03 & 2.73 & 2.44 \\
\hline
\end{tabular}

Table 3: Optimal PMU Placement Results for branch outage.

\begin{tabular}{c|c|c}
\hline System & IEEE-30 & IEEE-118 \\
\hline Branch & $(2,4)(2,6)(12,15)$ & $(4,5)(11,12)(17,30)(48,49)$ \\
outage & $(21,22)(23,24)$ & $(54,59)(56,59)(61,62)(70,74)$ \\
\hline & & $1,5,9,11,12,15,17,20,22,25$, \\
Optimal & $1,5,6,9,10,12$, & $\begin{array}{c}5,30,32,36,37,41,43,46,49, \\
\text { PMU }\end{array}$ \\
locations & $15,19,24,25,27$ & $\begin{array}{c}75,77,80,85,86,96,90,92,96, \\
100,105,110,115\end{array}$ \\
\hline No. PMUs & 11 & 40 \\
\hline
\end{tabular}

Simulations were also extended to PMU loss and optimal PMU placements considering ZIB (Table 4). The number of PMUs to assure a more robust monitoring system demands much more buses equipped with PMUs compared to normal conditions. Table 5 compares these results to other methods, showing good results in IEEE 14-bus and 24-bus networks but some less efficient results in the others (IEEE 30-bus, 57-bus and 118-bus).

\subsection{Optimization of Total Cost}

After validations of the first algorithm, simulations are also extended to total cost optimization problems in WAMS, and results are presented in this section. To be able to compare our results with related works, two cases (A and B) used in previous works are presented in Table 6, and used as input data for simulations. The same distance matrix is also assumed between buses from previous works, i.e., that all transmission lines have the same conductors 
Table 4: Optimal PMU Placement results for single PMU loss condition considering ZIB.

\begin{tabular}{|c|c|c|}
\hline System & Optimal PMU locations & $\begin{array}{l}\text { No. } \\
\text { PMUs }\end{array}$ \\
\hline IEEE-14 & $1,2,4,6,9,11,13$ & 7 \\
\hline IEEE-24 & $1,2,7,8,9,10,14,16,18,19,20,21$ & 12 \\
\hline IEEE-30 & $2,3,4,6,7,10,12,13,15,17,18,20,24,25,27,30$ & 16 \\
\hline IEEE-57 & $\begin{array}{c}2,3,5,7,9,10,12,15,17,19,21,23,27,28,31,35 \\
37,38,42,46,49,50,52,53,55,56,57\end{array}$ & 27 \\
\hline IEEE-118 & $\begin{array}{c}1,2,6,7,8,9,12,13,15,17,18,20,21,23,24,27 \\
28,29,32,34,36,37,40,41,44,45,46,49,51,52 \\
54,56,57,60,62,66,68,70,72,75,77,79,80,83 \\
85,86,87,89,90,92,94,96,100,102,105,107 \\
109,110,111,112,115,117,118\end{array}$ & 63 \\
\hline
\end{tabular}

Table 5: Comparison of optimal PMU placement results for single PMU loss condition considering ZIBs.

\begin{tabular}{lccccc}
\hline Methods & IEEE-14 & IEEE-24 & IEEE-30 & IEEE-57 & IEEE-118 \\
\hline Proposed & 7 & 12 & 16 & 27 & 63 \\
{$[\mathbf{2 8}]$} & 7 & 13 & 15 & 26 & 64 \\
{$[\mathbf{3 2}$} & 7 & N/A & 14 & 22 & 61 \\
\hline
\end{tabular}

with the same configurations and relative distances between system buses were extracted from the system admittance matrix 33. For each case, the total length of the transmission lines is shown in the same table.

\subsubsection{Case $A$}

Simulations for this case are summarized in Table 7, It can be noticed from these results that the optimal PMU configurations which result in lower costs and assure system observability require in general more PMUs when compared to simple OPP algorithms (Table 1). Additionally, having more PMUs is better for redundancy measurement and state estimation. This conclusion is also stated in previous works. However, when compared to related works in Table 8 
Table 6: Parameters considered in simulations.

\begin{tabular}{|c|c|c|}
\hline Parameters & Case A & Case B \\
\hline References & [4] & 5 \\
\hline $\begin{array}{c}\text { Network } \\
\text { Total } \\
\text { Distance, km }\end{array}$ & $\begin{array}{l}3.000 \quad \text { (IEEE-30), } \\
9.884 \text { (IEEE-118), }\end{array}$ & $\begin{array}{l}5.712 \text { (IEEE-57), } \\
25.129 \text { (IEEE-300) }\end{array}$ \\
\hline Unit costs & $\begin{array}{c}\text { PMU - } \$ 40.000 \\
\text { OPGW - } \$ 10.000 / \mathrm{km}\end{array}$ & $\begin{array}{l}\text { PMU - } \$ 40.000 \text { (with one voltage } \\
\text { and two current measuring } \\
\text { modules) } \$ 4000 \text { extra fee for any } \\
\text { additional current measurement } \\
\text { channel needed; OPGW - } \\
\$ 4.000 / \mathrm{km} \text {; switches - } \$ 4.000 ;\end{array}$ \\
\hline ZIB & No & Yes \\
\hline
\end{tabular}

our methodology shows a better efficiency in lowering meter planning cost in both IEEE 30-bus (-20.4\%) and IEEE 118-bus (-32.5\%). The resulting communication network is also smaller.

\subsubsection{Case B}

This case considers different cost structure for PMU and communication media. Simulations were done for normal operating conditions and PMU loss requirement and are presented in Tables 9 and 10 .

The results confirm previous arguments that the number of PMUs for a lower overall cost does not necessarily correspond to a minimal number of PMUs. Comparing these results to two related works with same simulation conditions (case B) in Table 11, it is possible to see that the proposed method is more effective to get a lower total cost for a meter planning system in IEEE 118-bus. The cost obtained by the algorithm managed to lower costs by $14.2 \%$ under constraint of normal operating condition and $20.5 \%$ for PMU loss requirement when compared to previous related works. Yet, the proposed algorithm obtained 
Table 7: Total Cost (PMU and CI) Optimization for Normal Operating Conditions - Case A.

\begin{tabular}{|c|c|c|c|c|c|}
\hline System & $\begin{array}{c}\text { No. } \\
\text { PMUs }\end{array}$ & PMUs & $\begin{array}{c}\text { No. } \\
\text { Switches }\end{array}$ & $\begin{array}{c}\mathbf{k m} \\
(\mathrm{OPGW})\end{array}$ & $\begin{array}{l}\text { US\$ } \\
\left(\mathrm{x} 10^{6}\right)\end{array}$ \\
\hline IEEE-14 & 5 & $4,5,6,7,9$ & 6 & 199 & 2.2 \\
\hline IEEE-30 & 10 & $\begin{array}{c}2,4,6,9,10,12,15 \\
20,25,27\end{array}$ & 11 & 625 & 6.7 \\
\hline IEEE-57 & 18 & $\begin{array}{c}1,4,9,15,18,21,24 \\
28,29,31,32,36,38 \\
\quad 39,41,46,51,54\end{array}$ & 35 & 1481.2 & 15.5 \\
\hline IEEE-118 & 36 & $\begin{array}{c}2,5,9,11,12,17,21, \\
27,30,31,32,34,37, \\
40,45,49,50,51,54, \\
59,65,66,68,70,71, \\
75,77,80,83,86,89 \\
92,96,100,105,110\end{array}$ & 51 & 1991.5 & 21.4 \\
\hline
\end{tabular}

more PMUs for normal conditions, which is better for measurements redundancy and state estimation process. The communication network and the number of nodes involved are smaller in our method.

Table 8: Comparison of results under normal conditions - Case A.

\begin{tabular}{|c|c|c|c|c|c|}
\hline System & Methods & $\begin{array}{l}\text { No. } \\
\text { PMUs }\end{array}$ & $\begin{array}{c}\text { No. } \\
\text { Switches }\end{array}$ & $\begin{array}{c}\mathbf{k m} \\
(\mathrm{OPGW})\end{array}$ & $\begin{array}{r}\mathrm{US} \$ \\
\mathrm{x} 10^{6}\end{array}$ \\
\hline \multirow{2}{*}{ IEEE-30 } & Proposed & 10 & 11 & 625 & 6.7 \\
\hline & 4 & 10 & 15 & 804.6 & 8.4 \\
\hline \multirow{2}{*}{ IEEE-118 } & Proposed & 36 & 51 & 1991.5 & 21.4 \\
\hline & 4 & 39 & 76 & 3012.6 & 31.7 \\
\hline
\end{tabular}


Table 9: Total Cost (PMU and CI) Optimization for Normal Operating Conditions, considering ZIB - Case B.

\begin{tabular}{|c|c|c|c|c|c|}
\hline System & $\begin{array}{l}\text { No. } \\
\text { PMUs }\end{array}$ & PMUs & $\begin{array}{c}\text { No. } \\
\text { Switches }\end{array}$ & $\begin{array}{c}\mathbf{k m} \\
(\mathrm{OPGW})\end{array}$ & $\begin{array}{r}\text { US\$ } \\
\left(\mathrm{x} 10^{6}\right)\end{array}$ \\
\hline IEEE-14 & 4 & $4,5,6,9$ & 5 & 199 & 1.0 \\
\hline IEEE-30 & 7 & $\begin{array}{c}2,4,10,12,15,20 \\
27\end{array}$ & 10 & 546.3 & 2.5 \\
\hline IEEE-57 & 16 & $\begin{array}{c}1,4,10,15,20,23 \\
28,29,31,32,36 \\
39,41,47,49,54\end{array}$ & 33 & 1476.7 & 6.5 \\
\hline IEEE-118 & 32 & $\begin{array}{c}3,8,11,12,17,21 \\
27,31,32,34,37 \\
40,45,49,51,54 \\
56,61,66,70,71 \\
75,77,80,83,86 \\
89,92,96,100 \\
105,110\end{array}$ & 49 & 1983.3 & 9.7 \\
\hline
\end{tabular}

\subsection{Optimization of Total Cost in Large Networks}

The algorithm was also tested on large networks, such as IEEE 300-bus and BR 5804-bus. Distances between buses in IEEE 300-bus were set following the same procedure stated for smaller IEEE networks in the previous section. For the larger Brazilian Network BR5804-bus the distance matrix was built in MATLAB@ using a random number generator $(r g n)$ seeded to a unit value and a randi function with mean equal to fourteen in order to get a total network length of $100.000 \mathrm{~km}$, which is near the real value [34. Results obtained in Table 12 have confirmed the influence of communication cost on resulting number of PMUs, which is in general higher for cost optimization problems than for simple minimization OPP approaches (Table 2).

It must be stated that different PMU and communication equipment costs could lead to different results. 
Table 10: Total Cost (PMU and CI) Optimization for PMU loss requirement, considering ZIB - Case B.

\begin{tabular}{|c|c|c|c|c|c|}
\hline System & $\begin{array}{c}\text { No. } \\
\text { PMUs }\end{array}$ & PMUs & $\begin{array}{c}\text { No. } \\
\text { Switches }\end{array}$ & $\begin{array}{c}\mathbf{k m} \\
(\mathrm{OPGW})\end{array}$ & $\begin{array}{r}\text { US\$ } \\
\left(\mathrm{x} 10^{6}\right)\end{array}$ \\
\hline IEEE-14 & 7 & $2,4,5,6,9,11,13$ & 8 & 376.7 & 1.8 \\
\hline IEEE-30 & 16 & $\begin{array}{c}2,3,4,6,7,10,12,13 \\
15,17,19,20,21,24,27 \\
30\end{array}$ & 19 & 1026.8 & 4.6 \\
\hline IEEE-57 & 30 & $\begin{array}{c}1,3,4,8,9,10,12,15 \\
19,20,22,24,27,29,30 \\
31,32,33,35,36,41,45 \\
46,47,49,51,53,54,56 \\
57\end{array}$ & 44 & 2347.4 & 10.3 \\
\hline IEEE-118 & 64 & $\begin{array}{c}1,3,5,6,8,9,11,12,15, \\
17,19,21,22,24,25,27, \\
29,31,32,34,36,37,40, \\
42,44,45,48,49,51,52, \\
54,56,57,59,62,65,66, \\
69,70,71,75,77,79,80, \\
83,85,86,87,89,90,92, \\
94,96,100,102,105,107, \\
109,110,111,112,115, \\
117,118\end{array}$ & 80 & 3016.0 & 14.5 \\
\hline
\end{tabular}

Table 11: Comparison of results in IEEE-118-bus - Case B.

\begin{tabular}{c|ccccc}
\hline $\begin{array}{c}\text { Operating } \\
\text { Conditions }\end{array}$ & Methods & $\begin{array}{c}\text { No. } \\
\text { PMUs }\end{array}$ & $\begin{array}{c}\text { No. } \\
\text { Switches }\end{array}$ & $\begin{array}{c}\text { km } \\
(\mathrm{OPGW})\end{array}$ & $\begin{array}{c}\text { US\$ } \\
(\mathrm{x} 10)^{6}\end{array}$ \\
\hline \multirow{3}{*}{ Normal } & Proposed & 32 & 49 & 1983.3 & 9.7 \\
& {$[5]$} & 30 & 60 & 2428.0 & 11.4 \\
& {$[\mathbf{1 9}$} & 30 & 58 & 2386.8 & 11.2 \\
\hline \multirow{2}{*}{ Loss of PMUs } & Proposed & 64 & 80 & 3016.0 & 14.5 \\
& {$[5]$} & 64 & 88 & 3799.7 & 18.5 \\
& {$[\mathbf{1 9}$} & 61 & 82 & 3706.2 & 18.0 \\
\hline
\end{tabular}


Table 12: Total cost optimization in large networks.

\begin{tabular}{ccccc}
\hline System & $\begin{array}{c}\text { No. } \\
\text { PMUs }\end{array}$ & $\begin{array}{c}\text { No. } \\
\text { Switches }\end{array}$ & $\begin{array}{c}\text { km } \\
(\mathrm{OPGW})\end{array}$ & $\begin{array}{c}\text { US\$ } \\
(\mathrm{x} 10)^{6}\end{array}$ \\
\hline IEEE-300 & 91 & 152 & 7862.4 & 35.9 \\
\hline BR-5804 & 2004 & 2887 & 18220 & 156.7 \\
\hline
\end{tabular}

\section{Conclusion}

A new methodology for total cost optimization in WAMS considering PMUs allocation and communication infrastructure costs was presented in this paper. Firstly, a simple optimal PMU placement algorithm based on Variable Neighbourhood Search metaheuristic was validated which has never been used before in related PMU placement problems.

This first algorithm proved to be flexible, efficient and scalable and in which a new graph theory metric proposed has played a fundamental role.

Subsequently, a total cost optimization problem was introduced and the previous algorithm was adapted and extended to solve this problem.

The algorithm proved its simplicity and flexibility in considering several operating conditions and real-life cost parameters for PMU and communication infrastructure. It was able to manage simulations in large and complex networks and resulted in good solutions compared to previous works.

\section{Acknowledgment}

This work was partially supported by CAPES-Project 88881.062156/201401, CNPq-Projects 462477/2014-2 and 304564/2016-8, FAPES-80607594/17NIDA Project (Brazil) and by the Foundation HEC Montréal (Canada). 


\section{References}

[1] R. B. Bobba, J. Dagle, E. Heine, H. Khurana, W. H. Sanders, P. Sauer, T. Yardley, Enhancing grid measurements: Wide area measurement systems, NASPInet, and security, IEEE Power and Energy Magazine 10 (1) (2012) 67-73.

[2] U. S. Department of Energy Office of Electricity, Factors affecting PMU installation costs, https://www.smartgrid.gov/files/ PMU-cost-study-final-10162014_1.pdf, access: 2018-06-21.

[3] R. Gore, M. Kande, Analysis of Wide Area Monitoring System architectures, in: Industrial Technology (ICIT), 2015 IEEE International Conference on, 2015, pp. 1269-1274. doi:10.1109/ICIT.2015.7125272.

[4] M. Shahraeini, M. S. Ghazizadeh, M. H. Javidi, Co-optimal placement of measurement devices and their related communication infrastructure in wide area measurement systems, IEEE Transactions on Smart Grid 3 (2) (2012) 684-691. doi:10.1109/TSG.2011.2178080

[5] M. B. Mohammadi, R. A. Hooshmand, F. H. Fesharaki, A new approach for optimal placement of PMUs and their required communication infrastructure in order to minimize the cost of the WAMS, IEEE Transactions on Smart Grid 7 (1) (2016) 84-93. doi:10.1109/TSG.2015.2404855.

[6] P. Kansal, A. Bose, Bandwidth and latency requirements for smart transmission grid applications, IEEE Transactions on Smart Grid 3 (3) (2012) $1344-1352$.

[7] P. Hansen, N. Mladenović, Variable neighborhood search, in: Search methodologies, Springer, 2014, pp. 313-337. 
[8] C.-S. Liao, T.-J. Hsieh, X.-C. Guo, J.-H. Liu, C.-C. Chu, Hybrid search for the optimal PMU placement problem on a power grid, European Journal of Operational Research 243 (3) (2015) 985-994.

[9] N. Meghanathan, Use of Centrality Metrics to Determine Connected Dominating Sets for Real-World Network Graphs , year=2015, pages $=243-248$, doi=10.1109/ITNG.2015.45, month=April,, in: Information Technology New Generations (ITNG), 2015 12th International Conference on.

[10] S. S. Noureen, V. Roy, S. B. Bayne, Phasor measurement unit integration: A review on optimal PMU placement methods in power system, in: Humanitarian Technology Conference (R10-HTC), 2017 IEEE Region 10, IEEE, 2017, pp. 328-332.

[11] R. Sodhi, S. Srivastava, S. Singh, Optimal PMU placement method for complete topological and numerical observability of power system, Electric Power Systems Research 80 (9) (2010) 1154-1159.

[12] G. N. Korres, N. M. Manousakis, T. C. Xygkis, J. Löfberg, Optimal phasor measurement unit placement for numerical observability in the presence of conventional measurements using semi-definite programming, IET Generation, Transmission \& Distribution 9 (15) (2015) 2427-2436.

[13] L. Huang, Y. Sun, J. Xu, W. Gao, J. Zhang, Z. Wu, Optimal PMU placement considering controlled islanding of power system, IEEE Transactions on Power Systems 29 (2) (2014) 742-755.

[14] M. Nazari-Heris, B. Mohammadi-Ivatloo, Application of heuristic algorithms to optimal PMU placement in electric power systems: An updated review, Renewable and Sustainable Energy Reviews 50 (2015) 214-228. 
[15] A. Monti, C. Muscas, F. Ponci, Phasor measurement units and wide area monitoring systems, Academic Press, 2016.

[16] A. Pal, C. Mishra, A. K. S. Vullikanti, S. Ravi, General optimal substation coverage algorithm for phasor measurement unit placement in practical systems, IET Generation, Transmission \& Distribution 11 (2) (2017) 347353.

[17] A. Pal, A. K. S. Vullikanti, S. Ravi, A PMU placement scheme considering realistic costs and modern trends in relaying, IEEE Transactions on Power Systems 32 (1) (2017) 552-561.

[18] R. Dubey, J. C. Muro, M. Popov, Cost Effective Wide Area Measurement Systems for Smart Power Network, IEEE Power and Energy Technology Systems Journal.

[19] S. P. Singh, S. P. Singh, Optimal cost wide area measurement system incorporating communication infrastructure, IET Generation, Transmission \& Distribution 11 (11) (2017) 2814-2821.

[20] Z. H. Rather, Z. Chen, P. Thgersen, P. Lund, B. Kirby, Realistic approach for phasor measurement unit placement: Consideration of practical hidden costs, IEEE Transactions on Power Delivery 30 (1) (2015) 3-15. doi: 10.1109/TPWRD.2014.2335059.

[21] B. Appasani, D. K. Mohanta, Co-Optimal Placement of PMUs and Their Communication Infrastructure for Minimization of Propagation Delay in the WAMS, IEEE Transactions on Industrial Informatics 14 (5) (2018) $2120-2132$.

[22] G. Caporossi, P. Hansen, Variable neighborhood search for extremal 
graphs. Three ways to automate finding conjectures, Discrete Mathematics 276 (1) (2004) 81-94.

[23] T. H. Cormen, Introduction to algorithms, MIT press, 2009.

[24] D. J. Brueni, L. S. Heath, The PMU placement problem, SIAM Journal on Discrete Mathematics 19 (3) (2005) 744-761.

[25] L. Ding, X. Gao, W. Wu, W. Lee, X. Zhu, D. Z. Du, Distributed Construction of Connected Dominating Sets with Minimum Routing Cost in Wireless Networks, in: Distributed Computing Systems (ICDCS), 2010 IEEE 30th International Conference on, 2010, pp. 448-457. doi:10.1109/ ICDCS.2010.17.

[26] E. Abiri, F. Rashidi, T. Niknam, M. R. Salehi, Optimal PMU placement method for complete topological observability of power system under various contingencies, International Journal of Electrical Power and Energy Systems 61 (2014) 585 - 593. doi:http://dx.doi.org/10.1016/j. ijepes.2014.03.068

[27] M. Hajian, A. M. Ranjbar, T. Amraee, B. Mozafari, Optimal placement of PMUs to maintain network observability using a modified BPSO algorithm, International Journal of Electrical Power \& Energy Systems 33 (1) (2011) $28-34$.

[28] B. S. Roy, A. Sinha, A. Pradhan, An optimal PMU placement technique for power system observability, International Journal of Electrical Power \& Energy Systems 42 (1) (2012) 71-77.

[29] T. L. Baldwin, L. Mili, M. B. Boisen, R. Adapa, Power system observability with minimal phasor measurement placement, IEEE Transactions on Power Systems 8 (2) (1993) 707-715. doi:10.1109/59.260810. 
[30] X. Liu, W. Wang, D. Kim, Z. Yang, A. O. Tokuta, Y. Jiang, The first constant factor approximation for minimum partial connected dominating set problem in growth-bounded graphs, Wireless Networks 22 (2) (2016) $553-562$.

[31] M. H. F. Wen, J. Xu, V. O. K. Li, Optimal multistage PMU placement for wide-area monitoring, IEEE Transactions on Power Systems 28 (4) (2013) 4134-4143. doi:10.1109/TPWRS.2013.2277741.

[32] K. G. Khajeh, E. Bashar, A. M. Rad, G. B. Gharehpetian, Integrated model considering effects of zero injection buses and conventional measurements on optimal PMU placement, IEEE Transactions on Smart Grid 8 (2) (2017) $1006-1013$.

[33] U. Washington, Power system test case archive, https://www.ee. washington.edu/research/pstca/, access: 2016-04-18.

[34] ONS, Power and Transmission system of Brazil, http://www.ons.org. br/pt/paginas/sobre-o-sin/mapas, access: 2016-04-18. 